\title{
Analyzing the Effectiveness of Policy Instruments on New Energy Vehicle Industry using Consistent Fuzzy Preference Relations
}

\section{A case study in China}

\author{
Dan $\mathrm{Li}^{1}$ Hongwei Guo ${ }^{* 1}$ Xianzhi Wang ${ }^{1}$ Ze Liu ${ }^{1}$ Cheng Li $^{1}$ Wuhong Wang ${ }^{1}$ \\ 1 Department of Transportation Engineering, Beijing Institute of Technology \\ *Corresponding Author, Email: guohongwei@bit.edu.cn
}

Received: 20 October, 2015; Accepted: 10 February, 2016

Key words: Sustainable development, New energy vehicles, Policy efficiency, Consistent fuzzy preference relations

\begin{abstract}
New energy vehicles (NEVs) have important implications for traffic pollution management and energy consumption. A series of industrial incentive policies have been introduced to promote the development of the NEV industry. However, the actual effects of the policies do not come up to expectations. Aiming to analyze the relationship between the policy instruments and the market acceptance of NEVs, a consistent fuzzy preference relations model is proposed to evaluate the efficiency of policies on the NEV industry. The model introduces an assessment criteria system with three criterions (industry development, technology research and development (R\&D), and NEV popularization). The quantitative weights of evaluation criteria are calculated by the fuzzy preference relation. The weights indicate the significance of the factors in the development of NEVs, especially when NEVs have not been widely accepted. With the proposed model, a case study in China shows that the policy of technology R\&D is the most crucial for the NEV industry in China. The policy of NEV industry development, on the other hand, is equally important. The results are hoped to give a better understanding of the relationship between government policy instruments and NEV development, the measures for enhancing the policy efficiency of NEV development.
\end{abstract}

\section{INTRODUCTION}

Nowadays, the concept of sustainable development with green economy, low-carbon travel and energy saving has become increasingly important and popular worldwide. It has been acknowledged that the adoption of NEVs is an effective way to reduce the dual pressures brought by energy crises and environmental protection agendas. New energy vehicles (NEVs) - vehicles using non-traditional fuels (ethanol, biogas, biodiesel) such as electric vehicles, fuel cell vehicles and hybrid electric vehicles - are of particular strategic importance for several reasons. To start with, reducing dependence on fossil fuels is one of the soundest reasons. Moreover, the debate on $\mathrm{CO}_{2}$ emissions and other air pollutants (vehicle emission is one of the major sources of air pollution) constitutes another reason. Realizing such challenges caused by the transportation sector, NEVs have become an inevitable choice 
for the sustainable development of the automobile industry in various countries. Several new propulsion measures for NEVs have emerged and already entered, or are ready to enter the market in the future. However, the development of the NEV industry does not perform up to expectations. Because of the cost disadvantages, limited driving range as well as inadequate charging facilities, the NEV industry has to overcome several obstacles in order to achieve a shift in the transportation sector. Therefore, government policy instruments are important to improve the technical level of NEVs and encourage the purchase of NEVs.

Many countries and regions, such as China, the United States and Europe, have set up goals to develop NEVs. Since 1990, the Chinese government has continuously enacted a series of regulations and support policies to promote the development of the NEV industry. In 2001, the Ministry of Science and Technology of the People's Republic of China (MOST) launched the '863 Program', a specialized electric vehicle (EV) project. It established three 'vertical' and three 'horizontal' schemes. During the last several years, China's NEV industry has begun to move from research towards commercialization and mass production. In 2008, the BYD dual-mode electric car F3DM won the approval of the Ministry of Industry and Information Technology (MIIT), which heralded a new stage of China's NEVs industrialization development. On January 24th, 2009, the Ministry of Finance with the Ministry of Science and Technology initiated the 'Ten Cities, Thousand Vehicles Program' to stimulate the adoption of NEVs (Zheng et al., 2012). In these cities, local governments encouraged using NEVs in public transportation, e.g. taxis, environmental sanitation vehicles, logistic vehicles, police vehicles, and postal vehicles. Meanwhile, an accompanying subsidy policy was also adopted to increase the sales of NEVs. By the end of 2010, most Chinese auto companies started mass production of own-brand NEVs, with many other Chinese firms investing in the industry of hybrid electric vehicles. In March 2013, the '12th Five-year Major National Innovation Base Construction Plan' put forward that new energy vehicles would be regarded as an area with high innovation and foundation advantages and be a pilot industry for the major national innovation base. In December, MIIT issued four national standards regarding electric vehicle charging interface and communication protocols, which was a huge step to perfecting China's NEVs standards system, impelling NEV pilot demonstration and promoting the coordinated development of NEVs.

In the United States, the Energy Policy Act of 1992 implemented an alternative fuel demonstration project, and identified mixtures containing 85 percent or more by volume of methanol with gasoline or other fuels as alternative fuels. In 2005, the U.S. Government decided to provide a \$2000 tax deduction for all qualifying hybrid vehicles. Nevertheless, the Energy Policy Act of 2005 replaced this tax deduction with a tax credit based on an individual vehicle's emissions profile and fuel efficiency compared to equivalent gasoline vehicles from January 2006 (Diamond, 2009). Credits vary from several hundred to several thousand dollars, and phase out over time after the manufacturer sells a total of 60,000 hybrid and lean-burn vehicles (Department of Energy, 2007). Many states offer additional incentives besides the federal tax credit. Starting in 2008, Colorado provided the most generous incentive structures with credits of $\$ 2500-\$ 6000$ according to the vehicle, while several other states offer incentives valued no less than $\$ 1500$ ( $\mathrm{Brad}$, 2010). Some states, e.g. New York, Virginia, Florida, California and Utah, allow hybrid owners to use high-occupancy vehicle lane access regardless of the number of occupants on one or more highways in the state. As of 
November 2014, about half the states have established incentives and tax or fee exemptions for BEVs and PHEVs (battery or plug-in EVs), or utility-rate breaks, and other non-monetary incentives such as free parking.

European governments are also making good efforts toward achieving high market penetration of NEVs. The goals of the individual countries are mostly similar - carbon-free fleets in pollution-free cities - many different approaches to making it happen are in place (Lindquist \& Wendt, 2011). In order to define the acceptable limits for exhaust emissions of new vehicles sold in EU member states, Europe legislates increasingly stringent emission standards. At the end of 2012, Euro VI emission regulations came into force. In 1995, Europe announced a green paper on its EU energy policy to actively promote diverse automotive energy and attach importance to developing hydrogen, natural gas and biofuels. In 2003, Europe released Future Prospects for European Hydrogen, and formulated the European Hydrogen Energy Roadmap. These measures forcefully promoted the industrialization and research and development (R\&D) of electric vehicles and enhanced the technological competitiveness of the automobile industry. In 2008, the EU passed legislative proposals on developing a new energy vehicles draft to further support the development of the clean-energy industry. At the same time, the European Parliament passed a bill that required public departments, enterprises and so forth to purchase fuel-efficient vehicles such as electric vehicles. In 2010, the European Commission brought forward a strategy to encourage the development of clean-energy vehicles (mainly referring to electric vehicles) and fuel-efficient vehicles. This was aimed at establishing a clean, energy saving transportation system, reducing the vehicle emission pollution and promoting the use of NEVs.

Nowadays, many scholars have carried out extensive research on the technology of NEVs, promoting the development of NEVs toward pure electric vehicles. Nevertheless, as a new emergent industry, the development of NEVs greatly depends on the support of government. For example, in China, the production and sales of NEVs are influenced significantly by government policy. However, there is still a huge gap between China's NEV industry and that of foreign countries on technology development, talent reserves and industrial cooperation.

Besides the NEV technology, previous research also focused on the policy instruments of the NEV industry. According to the literature review, policy guidance and planning played a vital role in the growth of the NEV industry (Yuan, Liu, \& Zuo, 2015). Stimulated by the policy shift, electric vehicle production has increased considerably, thereby contributing $23 \%$ and $44 \%$ of total NEV production in 2010 and 2011, respectively (Gong, Wang, \& Wang, 2013). Nevertheless, the NEV industry faces significant challenges related to the industrial chain, social factors and technologies. The Japanese Government has adopted a comprehensive strategy including R\&D, demonstration programs and market support guided by long-term strategic plans. The results show that flexibility, adaptability and cooperation in terms of technical choice is necessary in policy. Three alternative policy support measures, namely an up-front price support, a $\mathrm{CO}_{2}$ tax, and an increase in the fuel consumption tax for ICEs (internal combustion engines), could promote NEVs, and NEVs will be cost-competitive with ICEs if projected production volumes and thus economies of scale are reached (Gass, Schmidt, \& Schmid, 2014). Besides these alternative policy support measures, market support, even in the early phases of development, is an important complement to R\&D for gaining experience and building markets (يhman, 2006). Policy intending to give NEVs a foothold in the market should not only focus on mainstream 
consumers but also should focus on niche markets - especially car-sharing and postal fleets - and early adopters, including green consumers (Green, Skerlos, \& Winebrake, 2014). In China, the marketing strategy of enterprise and consumer behavior is influenced profoundly by government policy (Zhang, $X$. et al., 2013). Meanwhile, government policy has a positive adjustment function in the economic benefits of NEVs, consumers' purchase intentions and acceptable pricing. It further enhances the environmental protection spirit of consumers and meets their psychological needs. Government incentive policies, especially monetary incentives, impose a marked influence on the sales of NEVs. New technology developed by enterprises and research institutions under the encouragement of government could effectively reduce the cost of NEVs and make it more attractive (Liu \& Kokko, 2013). The consumers' willingness to buy NEVs and the purchase time is deeply affected by government policy (Zhang, Y., Yu, \& Zou, 2011).

The mentioned research mainly examined the effect of government policy through the NEV industry and consumer perspectives. However, it is important to analyze the effect of policy on the NEV industry from the perspective of the macro analysis of policy itself. With the aim of studying the effectiveness of policy instruments on the NEV industry and analyzing improvements for the policy efficiency of NEV development, this paper proposes an evaluation framework for NEV policy based on consistent fuzzy preference relations (CFPR). A case study of Chinese NEV policy evaluation is performed to verify the proposed method. The results are hoped to give a better understanding of NEV policy efficiency and policy measures for NEV development. The remaining part of the paper is organized as follows: Section 2 will introduce the consistent fuzzy preference relation as the evaluation method, Section 3 will propose the evaluating criteria for NEV policy efficiency, then, a case study in China is conducted to verify and validate the evaluation index and method, and finally, some conclusions and future works will be discussed in the last section.

\section{CONSISTENT FUZZY PREFERENCE RELATIONS}

Saaty (1980) proposed the analytic hierarchy process (AHP) for solving multi-criteria decision problems, which included many comparisons of criteria. In an AHP, a questionnaire needs to contain questions for every grouped $n$-criterion pairwise comparison. However, if the $n$ increases, or such $n$-criterion group increases, the number of pairwise comparisons also increases. This may cause experts mental fatigue or inconsistent interpretations due to so many questions and comparisons. In the case of inconsistencies, questionnaires have to be refilled again, which results in inefficiency and the wasting of time.

Consistent fuzzy preference relations (CFPR) can avoid the aforementioned problem effectively. CFPR is used to establish the pairwise comparison matrices and construct the decision matrices of pairwise comparisons using additive transitivity. Experts only need to answer n-1 comparisons. The remaining (n-1)(n/2-1) values of pairwise comparisons of each n-criterion can be derived by using the CFPR method, which only involves simple calculations, and the procedure guarantees a consistent result in comparisons. 


\subsection{Preference relations}

Preference relations (PR) enable experts to give values for a set of criteria and a set of alternatives. The value expresses the preference degree of the first alternative over the second alternative. Fundamentally, two kinds of preference relations are applied in the decision-making problems: multiplicative preference relations and fuzzy preference relations.

Multiplicative preference relations (Chiclana, Herrera, \& Herrera-Viedma, 1998): A multiplicative preference relation $A$ on a set of alternatives $X$ is represented by a matrix $\mathrm{A}$. A can be obtained by

$$
A \subseteq X \times X, A=\left(a_{i j}\right), \quad \forall i, j \in\{1, \mathrm{~K}, n\}
$$

where $a_{i j}$ is the preference ratio of alternative $x_{i}$ over $x_{j}$. Saaty (1980) suggests measuring $a_{i j}$ using a ratio scale from 1 to 9 scales. When $a_{i j}=1$ the indifference between $x_{i}$ and $x_{j} ; a_{i j}=9$ represents that $x_{i}$ is absolutely preferred to $x_{j} ; a_{i j}>1$ denotes that $x_{i}$ is preferred to $x_{j}$. The preference relation $A$ is typically assumed to be a multiplicative reciprocal, given by

$$
a_{i j} \cdot a_{j i}=1, \quad \forall i, j \in\{1, \mathrm{~K}, n\}
$$

Fuzzy preference relations (Chiclana, Herrera, \& Herrera-Viedma, 1998): a fuzzy preference relation $P$ on a set of alternatives $X$ is a fuzzy set denoted by the product set $X \times X$ with a membership function

$$
\mu_{p}: X \times X \rightarrow[0,1]
$$

The preference relation is represented by the $n \times n$ matrix, where $p_{i j}=\mu_{p}\left(\mathrm{x}_{i}, \mathrm{x}_{j}\right) \forall i, j \in\{1, \mathrm{~K}, n\}$. Herein, $p_{i j}$ indicates the fuzzy preference ratio of the alternative $x_{i}$ to $x_{j}$ when $p_{i j}=1 / 2$ indicates that no difference exists between $x_{i}$ and $x_{j}, p_{i j}=1$ means that $x_{i}$ is absolutely preferred to $x_{j}$, and $p_{i j}>1 / 2$ implies that $x_{i}$ is preferred to $x_{j}$. In this case, the fuzzy preference matrix $P$ is usually assumed to be an additive reciprocal, that is,

$$
p_{i j}+p_{j i}=1, \quad \forall i, j \in\{1, \mathrm{~K}, n\}
$$

However, inconsistency may exist in traditional decision matrices. To solve this problem, Herrera-Viedma et al. (2004) proposed that the consistent fuzzy preference relations (CFPR) could be used to construct the decision matrices of pairwise comparisons based on additive transitivity. Three important propositions in CFPR are described as follows.

\subsection{Consistent fuzzy preference relations}

Proposition 1. Suppose a set of alternatives, $X=\left\{x_{1}, \mathrm{~K}, x_{n}\right\}$, associated with a reciprocal multiplicative fuzzy preference relation $A=\left(a_{i j}\right)$ with $a_{i j} \in[1 / 9,9]$. Then, the corresponding reciprocal fuzzy preference relation, $P=\left(p_{i j}\right)$ with $p_{i j} \in[0,1]$ associated with $A$ is defined as $P=g(A)$, i.e.,

$$
p_{i j}=g\left(a_{i j}\right)=\frac{1}{2}\left(1+\log _{9} a_{i j}\right)
$$

where $g(*)$ is a transformation function which transforms a reciprocal multiplicative preference relation matrix into a preference relation. $\log _{9} a_{i j}$ is considered because $a_{i j}$ is between $1 / 9$ and 9 . When $a_{i j}$ is between $1 / 5$ and 5 , $\log _{5} a_{i j}$ is used.

Proposition 2. For a reciprocal fuzzy preference relation $P=g(A)$, where $P=\left(p_{i j}\right)$, the following statements are equivalent.

$$
\begin{array}{cc}
p_{i j}+p_{j k}+p_{k i}=\frac{3}{2}, & \forall i, j, k \\
p_{i j}+p_{j k}+p_{k i}=\frac{3}{2}, & \forall i<j<k
\end{array}
$$


Proposition 3. For a reciprocal fuzzy preference relation, $P=\left(p_{i j}\right)$, the following statements are equivalent.

$$
\begin{gathered}
p_{i j}+p_{j k}+p_{k i}=\frac{3}{2}, \quad \forall i<j<k \\
p_{i(i+1)}+p_{(i+1)(i+2)}+\mathrm{L}+p_{(j-1) j}+p_{j i}=\frac{j-i+1}{2}, \quad \forall i<j
\end{gathered}
$$

Following Proposition 3, we can structure a consistent fuzzy preference relation $P$ on $X=\left\{x_{1}, x_{2}, \mathrm{~K}, x_{n}, n \geq 2\right\}$ from $n-1$ preference values $\left\{p_{12}, p_{23}, \mathrm{~K}, p_{(n-1) n}\right\}$.

A decision matrix with values not in the interval $[0,1]$, but in an interval $[-k, 1+k], k>0$, can convert the obtained values with a transformation function that preserves reciprocity and additive consistency. The transformation function $f(\mathrm{x})$ is given in the following steps (Herrera-Viedma et al., 2004):

Step 1 Compute the set of preference values for B as

Step 2 Compute the value $k$

$$
\begin{aligned}
& B=\left\{p_{i j}, i<j \wedge p_{i j} \notin\left\{p_{12}, p_{23}, \mathrm{~K}, p_{(n-1) n}\right\}\right\} \\
& p_{i i}=\frac{J-\imath+1}{\hat{k}}-p_{i(i+1)}-p_{(i+1)(i+2)}-\mathrm{L}-p_{(i-1) i}
\end{aligned}
$$

$$
k=\left|\min \left\{B \mathrm{U}\left\{p_{12}, p_{23}, \mathrm{~K}, p_{(n-1) n}\right\}\right\}\right|
$$

Step 3

$$
p=\left\{p_{12}, \mathrm{~K}, p_{(n-1) n}\right\} \mathrm{U} B \mathrm{U}\left\{1-p_{12}, \mathrm{~K}, 1-p_{(n-1) n}\right\} \mathrm{U} \neg \mathrm{B}
$$

Step 4 The transformation function $f(x)$ is

$$
f:[-k, 1+k] \rightarrow[0,1], f(x)=\frac{x+k}{1+2 k}, k>0
$$

\subsection{Evaluation of the weights of criteria}

After the fuzzy preference relation matrices, $P=\left(p_{i j}\right)$, of pairwise comparisons is constructed, the weight of each factor is ready for calculation. The average preference, $f_{i}$, of each criterion in the main criteria can be computed by the following

$$
f_{i}=\frac{1}{n} \sum_{j=1}^{n} p_{i j}
$$

where $p_{i j}$ is the value in the preference relation matrix $P$ and $n$ is the number of criteria. The weight $w$ of each criterion can be defined as follows

$$
w_{i}=\frac{f_{i}}{\sum_{i=1}^{n} f_{i}}
$$

\subsection{Method discussions}

The CFPR method in the AHP hierarchy is more convenient than the traditional AHP method. Firstly, it is easier to compute the relative weights of each main criteria and sub-criteria using the CFPR method. The CFPR method does not need to consider any complex integration, differentiation or simultaneous equations. Secondly, the CFPR process enables researchers to effectively reduce the pairwise comparison frequency. For instance, the CFPR 
method only needs to perform $2+7+5+4=18$ pairwise comparisons, whereas the AHP method must perform $\mathrm{C}_{2}^{3}+\mathrm{C}_{2}^{8}+\mathrm{C}_{2}^{6}+\mathrm{C}_{2}^{5}=56$ pairwise comparisons. And obviously, the CFPR method spends less time in comparison than the AHP method. Thirdly, the CFPR method ensures consistency. Inconsistency always occurs during comparison with traditional AHP if each group has a number of criteria to compare. Nevertheless, the consistency of decision matrices can be guaranteed by CFPR.

\section{CRITERIA FOR EVALUATING NEV POLICY}

New energy vehicle policy, as a public policy, is crucial to consider in the process of designing an evaluation system for NEV policy. NEV policy is a complex system and a large number of factors need to be considered before the evaluation. Whether the evaluation system is reasonable or not will determine the result of the evaluation directly. In the premise of following the principle of comprehensiveness, comparability, independence, guidance and quantification, great attention is paid to guarantee the clarity of the criteria selection and the gradation of the criteria structure, and to establish a perfect, scientific and reasonable evaluation system. According to the general procedure for public policy making, such as policy formulation and implementation, a NEV policy evaluation criteria system is established. It contains the following three aspects: NEV industry development criterion, NEV technology research and development criterion, as well as NEV application and popularization criterion.

The Criterion A (NEV industry development criterion) mainly focuses on industry development policy, which is the base of NEV industry development. The scientific, feasibility and comprehensiveness of the industry development policy provides a good condition for the healthy development of the NEV industry. The Criterion A contains eight sub-criteria as follows (see Table 1 below). The standard system includes battery specification, charging station (pile), standards of charging facilities, standard of key components, etc. The access system supports social capital and companies with technology innovation capability to develop and manufacture NEVs (Brown, Pyke, \& Steenhof, 2010). An industry development roadmap provides a development orientation for the NEV industry. An industrial R\&D subsidy is necessary to supply effective capital support for companies and is significantly and positively correlated to a country's NEV market share (Sierzchula et al., 2014). NEV infrastructures contain charging stations, charging piles, parking lots, etc. Infrastructures are very important for the development of the NEV industry because NEV industrialization needs the support of infrastructure.

The Criterion B (NEV technology research and development criterion) pertains to the core technology of NEVs. The core technology of NEVs, including the technology of key parts and the technology of related infrastructure, is a vital factor that constrains the development of the NEV market. Only technical breakthroughs can drive ordinary consumers to purchase NEVs. The Criterion B has six sub-criteria demonstrated as follows (see Table 1). A charging station (pile) is the charging place for NEVs when the battery power is low. The charging time in express charge mode and slow charge mode are two of the most important factors influencing consumer decisions to buy NEVs. Battery technology determines battery energy storage ability and service life, which affects NEV mileage. About the NEV power system, there are several factors that should be taken into consideration, e.g. the type of NEV, charging time, charging characteristic and charging mode. 
With the development of NEV technology, it is necessary to establish a convenient integration power system to efficiently manage the power supply process in generation, transmission and distribution. Therefore, other new technology, such as intelligent transportation technology and connected vehicles, also play an important role in the development of the NEV industry.

The Criterion C (NEV application and popularization criterion) mostly concerns the widespread use of NEVs. For now, most consumers adopt a waitand-see attitude to NEVs. The application and popularization of NEVs is an effective strategy to eliminate the wait-and-see attitude of consumers toward buying NEVs. The Criterion $\mathrm{C}$ includes five sub-criteria illustrated as follows (see Table 1). Demonstration effect means drawing the attention of the consumer through presenting NEVs as taxis, buses, postal vehicles and so on (Zheng et al., 2012). Consumer subsidy is a general way to encourage consumers to purchase NEVs. The subsidy standards always differ depending on driving mileage. Consumer cognition and acceptance levels reflect consumers' preferences for NEVs, which is most likely to affect consumers' choice for NEVs (Zhang, Y., Yu, \& Zou, 2011). Government procurement is an effective manner to promote NEVs when NEVs are not widely accepted by consumers.

The main criteria and the sub-criteria for evaluating NEV policy are listed in Table 1.

Table 1 The AHP model of New Energy Vehicle Industry Policy

\begin{tabular}{|c|c|c|c|}
\hline Notation & Main criteria (Level 1) & Notation & Sub-criteria (Level 2) \\
\hline \multirow[t]{8}{*}{$\overline{\mathrm{A}}$} & $\begin{array}{l}\text { NEV industry } \\
\text { development criterion }\end{array}$ & $\overline{\mathrm{A} 1}$ & $\begin{array}{l}\text { industry standard system and access } \\
\text { system }\end{array}$ \\
\hline & & $\mathrm{A} 2$ & $\begin{array}{l}\text { technological achievements } \\
\text { industrialization and marketization }\end{array}$ \\
\hline & & A3 & industry development roadmap \\
\hline & & A4 & energy consumption structure \\
\hline & & A5 & industrial tax preference \\
\hline & & A6 & industrial R\&D subsidy \\
\hline & & A7 & infrastructure \\
\hline & & A8 & business model \\
\hline \multirow[t]{6}{*}{ B } & NEV technology research & B1 & charging station (pile) technology \\
\hline & and development & B2 & technical level of total vehicle \\
\hline & criterion & B3 & battery technology \\
\hline & & B4 & power system technology \\
\hline & & B5 & intelligent technology \\
\hline & & B6 & connected vehicle technology \\
\hline \multirow[t]{6}{*}{$\mathrm{C}$} & NEV application and & $\mathrm{C} 1$ & demonstration effect \\
\hline & popularization criterion & $\mathrm{C} 2$ & consumer tax preference \\
\hline & & $\mathrm{C} 3$ & subsidy for NEV purchase \\
\hline & & $\mathrm{C} 4$ & consumer cognitive and acceptable \\
\hline & & & level \\
\hline & & $\mathrm{C} 5$ & government procurement \\
\hline
\end{tabular}




\section{CASE STUDY}

This paper summarized some influential factors according to the AHP hierarchy mentioned in aforementioned literature. The sample data were obtained from six NEV policy experts. In this example, the entire procedure for constructing a decision matrix is shown as follows:

Step 1 According to Proposition 1, the results of the experts' scores, expressed by linguistic variables, are transferred into Saaty's scale as listed in Table 2 (below) for all the 22 criteria. Tables 3-6 show the experts' scores.

Step 2 Use Eq. (5) to transfer each expert's scores into raw scores and get the initial decision matrices. The rest of the decision matrices values can be obtained by using Propositions 1 and 3 .

Step 3 Taking into account each expert's views, compute the average fuzzy preference relation of each expert and regard it as the basis of calculating each criterion weight. The complete decision matrices of main criteria and sub-criteria are summarized in Tables 7-10.

Step 4 By using Eq. (10) and Eq. (11), the average preference and the weight of every criterion and sub-criteria can be acquired. The results are shown in the columns 'average' and 'weight' in Tables 7-10 respectively. The column 'rank' in Tables 7-10 represents the degree of preference. The larger the weight, the higher rank.

Table 2 Degree of relative importance between two criteria

\begin{tabular}{cc}
\hline Definition & Degree of relative importance \\
\hline Equally important & 1 \\
Moderately important & 3 \\
Strongly important & 5 \\
Very strongly important & 7 \\
Absolutely important & 9 \\
\hline
\end{tabular}

Table 3 Preference relation matrix for pairwise comparison of main criteria

\begin{tabular}{ccccccc}
\hline & E1 & E2 & E3 & E4 & E5 & E6 \\
\hline A & $1 / 3$ & $1 / 3$ & 1 & $1 / 5$ & $1 / 3$ & 1 \\
$\mathrm{~B}$ & 3 & 5 & 3 & 3 & 5 & 3 \\
$\mathrm{C}$ & & & & & & \\
\hline
\end{tabular}

Table 4 Preference relation matrix for pairwise comparison of Criterion $\mathrm{A}$

\begin{tabular}{ccccccc}
\hline \multicolumn{1}{c}{} & E1 & E2 & E3 & E4 & E5 & E6 \\
\hline A1 & $1 / 3$ & $1 / 3$ & $1 / 5$ & $1 / 5$ & 1 & $1 / 3$ \\
A2 & 3 & 5 & 5 & 7 & 3 & 5 \\
A3 & $1 / 3$ & $1 / 7$ & $1 / 3$ & $1 / 5$ & $1 / 5$ & $1 / 3$ \\
A4 & 3 & 3 & 3 & 3 & 5 & 3 \\
A5 & 1 & 3 & 3 & 3 & 1 & 3 \\
A6 & $1 / 5$ & $1 / 3$ & $1 / 5$ & $1 / 3$ & $1 / 3$ & $1 / 5$ \\
A7 & 7 & 7 & 5 & 5 & 7 & 5 \\
A8 & & & & & & \\
\hline
\end{tabular}

Table 5 Preference relation matrix for pairwise comparison of Criterion B

\begin{tabular}{ccccccc}
\hline & E1 & E2 & E3 & E4 & E5 & E6 \\
\hline B1 & 3 & 5 & 5 & 7 & 5 & 3 \\
B2 & $1 / 5$ & $1 / 3$ & $1 / 3$ & $1 / 3$ & $1 / 7$ & $1 / 5$ \\
B3 & 3 & 5 & 5 & 3 & 3 & 5 \\
B4 & 3 & 3 & 3 & 7 & 5 & 3 \\
B5 & 1 & 3 & 3 & 5 & 3 & 1 \\
\hline
\end{tabular}

Table 6 Preference relation matrix for pairwise comparison of Criterion $\mathrm{C}$

\begin{tabular}{lllllll}
\hline & E1 & E2 & E3 & E4 & E5 & E6 \\
\hline $\mathrm{C} 1$ & 3 & 5 & 5 & 3 & 5 & 3 \\
$\mathrm{C} 2$ & 1 & $1 / 3$ & 1 & $1 / 3$ & $1 / 3$ & 1
\end{tabular}




\begin{tabular}{lllllll} 
C3 & $1 / 3$ & $1 / 5$ & $1 / 7$ & $1 / 5$ & $1 / 3$ & $1 / 3$ \\
$\mathrm{C} 4$ & 5 & 7 & 3 & 5 & 5 & 3 \\
$\mathrm{C} 5$ & & & & & & \\
\hline
\end{tabular}

Table 7 Complete comparison matrix and rank of main criteria

\begin{tabular}{lllllll}
\hline & A & B & C & Average & Weight & Rank \\
\hline A & 0.500 & 0.357 & 0.653 & 0.503 & 0.335 & 2 \\
B & 0.643 & 0.500 & 0.796 & 0.646 & 0.431 & 1 \\
C & 0.347 & 0.204 & 0.500 & 0.351 & 0.234 & 3 \\
\hline
\end{tabular}

Table 8 Complete comparison matrix and rank of Criterion A

\begin{tabular}{cccccccccccc}
\hline & A1 & A2 & A3 & A4 & A5 & A6 & A7 & A8 & Average & Weight & Rank \\
\hline A1 & 0.500 & 0.331 & 0.615 & 0.364 & 0.587 & 0.744 & 0.499 & 0.831 & 0.559 & 0.140 & 3 \\
A2 & 0.669 & 0.500 & 0.785 & 0.534 & 0.756 & 0.913 & 0.669 & 0.999 & 0.728 & 0.182 & 1 \\
A3 & 0.385 & 0.215 & 0.500 & 0.249 & 0.471 & 0.628 & 0.384 & 0.715 & 0.443 & 0.111 & 6 \\
A4 & 0.636 & 0.466 & 0.751 & 0.500 & 0.723 & 0.879 & 0.635 & 0.966 & 0.695 & 0.174 & 2 \\
A5 & 0.413 & 0.244 & 0.529 & 0.277 & 0.500 & 0.657 & 0.412 & 0.744 & 0.472 & 0.118 & 5 \\
A6 & 0.256 & 0.087 & 0.372 & 0.121 & 0.343 & 0.500 & 0.256 & 0.587 & 0.315 & 0.079 & 7 \\
A7 & 0.501 & 0.331 & 0.616 & 0.365 & 0.588 & 0.744 & 0.500 & 0.831 & 0.560 & 0.140 & 3 \\
A8 & 0.169 & 0.001 & 0.285 & 0.034 & 0.256 & 0.413 & 0.169 & 0.500 & 0.228 & 0.057 & 8 \\
\hline
\end{tabular}

Table 9 Complete comparison matrix and rank of Criterion B

\begin{tabular}{cccccccccc}
\hline & B1 & B2 & B3 & B4 & B5 & B6 & Average & Weight & Rank \\
\hline B1 & 0.500 & 0.696 & 0.523 & 0.699 & 0.875 & 0.999 & 0.716 & 0.239 & 1 \\
B2 & 0.304 & 0.500 & 0.327 & 0.504 & 0.680 & 0.804 & 0.520 & 0.173 & 3 \\
B3 & 0.477 & 0.673 & 0.500 & 0.676 & 0.852 & 0.977 & 0.692 & 0.231 & 2 \\
B4 & 0.301 & 0.496 & 0.324 & 0.500 & 0.676 & 0.801 & 0.516 & 0.172 & 4 \\
B5 & 0.125 & 0.320 & 0.148 & 0.324 & 0.500 & 0.625 & 0.340 & 0.113 & 5 \\
B6 & 0.001 & 0.196 & 0.023 & 0.199 & 0.375 & 0.500 & 0.216 & 0.072 & 6 \\
\hline
\end{tabular}

Table 10 Complete comparison matrix and rank of Criterion $\mathrm{C}$

\begin{tabular}{ccccccccc}
\hline & C1 & C2 & C3 & C4 & C5 & Average & Weight & Rank \\
\hline C1 & 0.500 & 0.815 & 0.723 & 0.414 & 0.765 & 0.643 & 0.257 & 2 \\
C2 & 0.185 & 0.500 & 0.408 & 0.099 & 0.449 & 0.328 & 0.131 & 5 \\
C3 & 0.277 & 0.592 & 0.500 & 0.191 & 0.541 & 0.420 & 0.168 & 3 \\
C4 & 0.586 & 0.901 & 0.809 & 0.500 & 0.851 & 0.729 & 0.292 & 1 \\
C5 & 0.235 & 0.551 & 0.459 & 0.149 & 0.500 & 0.379 & 0.152 & 4 \\
\hline
\end{tabular}

Table 7 shows that the rank of main criteria is B (NEV technology research and development criterion) $>$ A (NEV industry development criterion) $>\mathrm{C}$ (NEV application and popularization criterion), which reveals the opinions of experts on NEV policy. The result indicates that NEV technology research and development criterion is the most important factor in NEV policy. NEV technology includes battery technology, power system technology, charging technology, etc. Technology research and development is the core issue for the NEV industry and the foundation for its development. Hence, NEV policy should pay more attention to technology research and development of NEVs. NEV industry development criterion is another key criterion. Many countries in the world take NEV industry development into account as a part of a national strategy, establishing and implementing incentive policies to support NEV R\&D. The difference in NEV R\&D policy between various countries is the priority of the research area. For instance, Japan makes industrial competitiveness its first target. Therefore, Japan focuses on developing three kinds of electric vehicle: Plug-in Hybrid, Pure Electric and Fuel Cell. The United States pays more attention to energy conservation and emissions reduction. As a result, Extended Range Electric Vehicles and Pure Electric Vehicles are given priority in the process of industry development.

In the sub-criteria of NEV industry development criterion (Criterion A), $\mathrm{A} 2>\mathrm{A} 4>\mathrm{A} 1=\mathrm{A} 7>\mathrm{A} 5>\mathrm{A} 3>\mathrm{A} 6>\mathrm{A} 8$ (corresponding respectively to 
technological achievements in industrialization and marketization, improving energy consumption structure, industry standard system and access system, infrastructure, industrial tax preference, industry development roadmap, industrial R\&D subsidy, and business model) as shown in Table 8. Industrialization and marketization of technological achievements has the biggest impact. Research of NEVs is conducted in universities and scientific research institutions. Most results of previous research remain in the laboratory stage, which lacks industrialization, particularly regarding the power system, battery and other key parts. The result demonstrates that policy has to encourage companies and research institutes to use new technology to improve the performance of NEVs, which is an important factor to expand the NEV market. Improving energy consumption structures requires reducing dependence on petroleum resources and increasing the use of solar energy, wind energy, hydropower and other renewable energy sources. Moreover, improving the consciousness of the people, to encourage them to use green energy is also a vital step. Policy makers should focus on providing infrastructure for the large-scale production of NEVs. In addition, since infrastructure is strongly related to NEV ownership, different kinds of infrastructure should be provided according to different usage modes and it should be made sure that the speed of infrastructure construction and NEVs popularization are consistent (Sierzchula et al., 2014). Other criteria such as industry standard systems and access systems, industry development roadmaps and industrial tax preferences are also situated in relatively upper ranks cannot be ignored when formulating policies.

According to Table 9, the rank of the sub-criteria of NEV technology research and development criterion (Criterion B) is $\mathrm{B} 1>\mathrm{B} 3>\mathrm{B} 2>\mathrm{B} 4>\mathrm{B} 5>$ B6 (corresponding respectively to charging station (pile) technology, battery technology, technical level of total vehicle, power system technology, intelligent technology, connected vehicle technology). According to the result, charging station (pile) technology ranks the top. The charging station (pile) technology is closely related to charge time, construction cost, etc. Consumers want a charge time as short as possible, as well as to charge their vehicles anytime and anywhere. Battery technology of NEVs is another important criterion because it determines the driving mileage of NEVs. The battery technology is the most crucial criterion that consumers always take into consideration, as it is the evaluating standard when buying an NEV. Improving whole vehicle technology could shorten product development periods, reduce costs and enhance competitiveness. It is beneficial to improve NEV industry competitiveness. The other three criterions, power system technology, intelligent technology and vehicular networking technology, are also critical for NEV technology research and development.

\section{CONCLUSIONS}

In this paper, the consistent fuzzy preference relations (CFPR) are introduced to evaluate the efficiency in NEV policy, which includes three main criteria. According to CFPR in AHP structures, it is easy to construct the multi-criteria decision matrices and to evaluate the significance of each criterion of NEV policy. The policy efficiency can be directly evaluated with the weights calculated using expert scores. The process of calculation is easy and simple. Important information can be acquired from the evaluation results for use by policy makers in future practice. According to the weights in the proposed model, the most important main criterion is the NEV technology 
research and development criterion. Nowadays, NEVs are limited by disadvantages such as battery capacity and driving range. Many key technologies of NEVs, especially power battery technology, fuel cell technology and hybrid energy management systems, are technical difficulties all over the world. It is difficult to achieve a breakthrough in a short time, hence policy makers need to introduce policies focusing on technology R\&D to encourage universities, research institutions and enterprises to overcome technical obstacles. Certainly, the other two main criterions should also receive enough attention.

In the sub-criteria, charging station (pile) technology, battery technology of NEVs, technological achievements for industrialization and marketization, improving energy consumption structure, infrastructure, consumer cognition and acceptance levels, demonstration effect and consumer subsidies exert an important influence on the growth of the NEV industry. These criteria constitute aspects such as technology, marketing, consumption, infrastructure and subsidies. However, whether consumers accept NEVs still depends on the economy of NEVs, the convenience of infrastructure and services, encouragement policies, as well as the cognition of consumers. Policy makers should consider these aspects when formulating policies. The development and popularization of NEVs needs powerful support from the government. In this paper, as an example, only several professionals' opinions were collected to estimate and verify the model. In reality, NEV user perspectives are important to improve NEV technology effectiveness, and their preferences are useful to estimate the model. Therefore, the data sample will be enlarged in future work to obtain more instructive results.

\section{ACKNOWLEDGEMENTS}

This research was supported in part by the National Nature Science Foundation of China under grant 71301010, 51378062, Basic research foundation of Beijing Institute of Technology (No. 20150342010).

\section{REFERENCES}

Åhman, M. (2006). "Government Policy and the Development of Electric Vehicles in Japan". Energy Policy, 34(4), 433-443.

Brad, B. (2010). "Hybrid and Plug-in Incentives and Rebates - Region by Region". Retrieved from http://www.hybridcars.com/region-by-region on 08/03, 2010.

Brown, S., Pyke, D., \& Steenhof, P. (2010). "Electric Vehicles: The Role and Importance of Standards in an Emerging Market". Energy Policy, 38(7), 3797-3806.

Chiclana, F., Herrera, F., \& Herrera-Viedma, E. (1998). "Integrating Three Representation Models in Fuzzy Multipurpose Decision Making Based on Fuzzy Preference Relations". Fuzzy sets and Systems, 97(1), 33-48.

Department of Energy. (2007). "New Energy Tax Credits for Hybrids".

Diamond, D. (2009). "The Impact of Government Incentives for Hybrid-Electric Vehicles: Evidence from Us States". Energy Policy, 37(3), 972-983.

Gass, V., Schmidt, J., \& Schmid, E. (2014). "Analysis of Alternative Policy Instruments to Promote Electric Vehicles in Austria". Renewable Energy, 61, 96-101.

Gong, H., Wang, M. Q., \& Wang, H. (2013). "New Energy Vehicles in China: Policies, Demonstration, and Progress". Mitigation and Adaptation Strategies for Global Change, 18(2), 207-228.

Green, E. H., Skerlos, S. J., \& Winebrake, J. J. (2014). "Increasing Electric Vehicle Policy Efficiency and Effectiveness by Reducing Mainstream Market Bias". Energy Policy, 65, $562-566$. 
Herrera-Viedma, E., Herrera, F., Chiclana, F., \& Luque, M. (2004). "Some Issues on Consistency of Fuzzy Preference Relations". European Journal of Operational Research, 154(1), 98-109.

Lindquist, K., \& Wendt, M. (2011). "Electric Vehicle Policies, Fleet, and Infrastructure: Synthesis". (In Doyle, J. (Ed.), Transportation Synthesis Report: Public Private Partnerships Division Washington State Department of Transportation.

Liu, Y., \& Kokko, A. (2013). "Who Does What in China's New Energy Vehicle Industry?". Energy Policy, 57, 21-29.

Saaty, T. L. (1980). The Analytic Hierarchy Process: Planning, Priority Setting, Resources Allocation. New York: McGraw.

Sierzchula, W., Bakker, S., Maat, K., \& van Wee, B. (2014). "The Influence of Financial Incentives and Other Socio-Economic Factors on Electric Vehicle Adoption". Energy Policy, 68, 183-194.

Yuan, X., Liu, X., \& Zuo, J. (2015). "The Development of New Energy Vehicles for a Sustainable Future: A Review". Renewable and Sustainable Energy Reviews, 42, 298-305.

Zhang, X., Wang, K., Hao, Y., Fan, J., \& Wei, Y. (2013). "The Impact of Government Policy on Preference for Nevs: The Evidence from China". Energy Policy, 61, 382-393.

Zhang, Y., Yu, Y., \& Zou, B. (2011). "Analyzing Public Awareness and Acceptance of Alternative Fuel Vehicles in China: The Case of Ev". Energy Policy, 39(11), 7015-7024.

Zheng, J., Mehndiratta, S., Guo, J. Y., \& Liu, Z. (2012). "Strategic Policies and Demonstration Program of Electric Vehicle in China". Transport Policy, 19(1), 17-25. 